\title{
Three-Element Fractional-Order Viscoelastic Arterial Windkessel Model
}

\author{
Mohamed A. Bahloul Member, IEEE and Taous Meriem Laleg-Kirati Senior Member, IEEE
}

\begin{abstract}
Arterial hemodynamic assessment has always been essential for clinical Cardiovascular System (CVS) diagnosis. Using Windkessel (WK) lumped parametric model as noninvasive measurement tool provides the potential of achieving a very convenient, computational inexpensive and accurate prediction of the arterial parameters. Many versions of WK models have been proposed and extensively studied, over the last century. In general, they can be classified into two categories: elastic and viscoelastic models. Recently, several studies have discussed the potential of describing the arterial wall viscoelasticity using fractional order models, reducing the number of parameters and exposing a natural response. Hence, a key missing item in the arterial Windkessel modeling is a fractionalorder analog component that can provide a reliable, realistic and reduced representation of the fractional viscoelasticity behavior. In this paper, we present, for the first time, a three-element fractional-order viscoelastic Windkessel model. The proposed model incorporates a fractional-order capacitor that substitutes the ideal capacitor of standard three-elements WK model. The latter non-ideal element combines both resistive and capacitive properties which displays viscoelastic behavior of the arterial vessel. The contribution of both properties is controlled by the fractional differentiation order $\alpha$ enabling an accurate and reliable physiological description.
\end{abstract}

Keywords-Arterial hemodynamic, Arterial viscoelasticity, Lumped parameter model, Fractional-order model, Fractional order capacitor.

\section{INTRODUCTION}

M Odeling the arterial hemodynamic has been considered as an essential tool for the Cardiovascular System (CVS) assessment and diagnosis. It also has a paramount importance in the study of physio-pathological symptoms and diseases affecting the systemic arterial system [1]. Hence, many research studies have been invested for the sake of modeling and characterization of the arterial system parameters such as: 1) Aortic characteristic impedance, 2) Arterial compliance and 3) Arterial peripheral resistance [2]. Since the pioneer Frank's arterial model, Windkessel (WK) lumped parameters models have shown to be good candidates to successfully characterize the systemic hemodynamic in a simple and comprehensive way. In fact, WK models represent a powerful, non-invasive tool that help to evaluate the vascular features [3]. Many WK models have been proposed and extensively studied. In general, they can be classified into elastic and viscoelastic models. Elastic WK models consider the mechanical behaviors of the vessel's

The authors are with Computer, Electrical and Mathematical Sciences and Engineering Division (CEMSE), King Abdullah University of Science and Technology (KAUST), Thuwal 23955-6900, Makkah Province, Saudi Arabia. E-mail:\{mohamad.bahloul, taousmeriem.laleg\}@ kaust.edu.sa wall purely elastic and represented by an ideal capacitor. However, Viscoelastic Windkessel (VWK) models suppose the blood vessel motions to exhibit both elastic and viscous properties [4]. In addition, several studies have claimed that similar to most biological tissues CVS wall material shows a viscoelastic behavior rather than pure elastic behavior. Indeed, arteries are considered as a viscoelastic reservoir. They are modeled as a superposition of viscous and elastic components where the transmission of the mechanical energy might be split into two modules: the first module represents the stored energy into wall tissues which can be retrieved due to elasticity capabilities, while the second module refers to the dissipated energy caused by the viscous property. The deep understanding of the blood vessel viscoelasticity and the knowledge of its variety effect are imperative, in the study of degenerative diseases that affect arterial network [5], [6]. Recently, many studies have shown that the arterial wall motion can be described using fractional-order models consisting of fractional differential equations, thus reducing the number of parameters and showing a natural response [7]-[11]. They have interesting properties that help in modeling accurately complex systems including biological systems. In the present study, we propose a fractional-order Viscoelastic Windkessel Model, for the first time to the best of our knowledge. Similar to the standard three-element Windkessel (3-WK), the proposed model characterizes the aortic characteristic impedance $Z_{C}$ and the total peripheral resistance $R_{p}$ by ideal resistors. However, we substitute the ideal capacitor of 3-WK by a fractional-order capacitor $C_{F}$ (Constant phase Element). The latter non-ideal component combines both resistive and capacitive properties which displays the fractional viscoelastic behavior of the arterial vessel. The contribution of both properties is controlled by the fractional differentiation order $\alpha$ enabling an accurate and reliable physiological description. We study the effect of varying the fractional differentiation order value $\alpha$ on the complex and frequency dependent arterial compliance and arterial stiffness. We also examine the effect of $\alpha$ values on the modulus and phase angle of the aortic input impedance. The rest of the paper is organized as follows. Section II introduces preliminary concepts related to this work and describes the fractional calculus applied to Arterial viscoelasticity. In Section III, the proposed model derivations are shown. Section IV presents the numerical simulations of the proposed model and further discussion. 


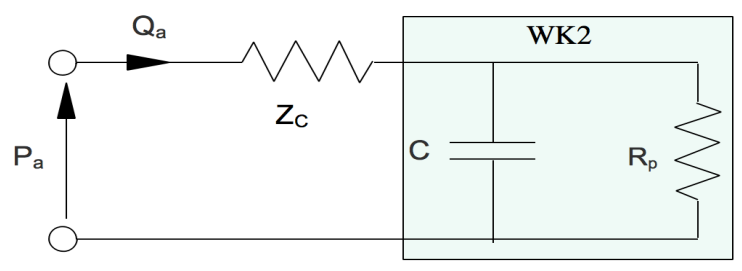

Fig. 1: Three Element Windkessel model analog circuit. This model consists of a resistance $Z_{C}$ representing the characteristic aortic impedance in series with the two element Windkessel model that comprises an ideal capacitor $C$ connected to a resistor $R_{p}$ which exhibit the total arterial compliance and peripheral resistance respectively.

Finally, Section V presents the conclusion and future perspectives on potential applications of the proposed model.

\section{MOTIVATION AND BACKGROUND}

\section{A. Windkessel lumped parameter arterial modeling}

Windkessel lumped parameter model is the most commonly used 0-Dimension method (No space variation) that describes the systemic arterial system function of the threeelementary analog device components: resistor, capacitor and inductor. WK models present the arterial hemodynamic by linking the blood flow, and blood pressure to the arterial resistance and compliance [3] [12]. It is formulated based on the analogy between physiological, hydraulic and electrical systems [4].

The first two element Windkessel (2-WK) model was proposed by Otto Frank in 1899. It consists of a resistor representing the total arterial peripheral resistance connected in parallel to an ideal capacitor, which characterizes the total vessel compliance. Whereas, the WK2 is very simple, it does not produce the real systemic input impedance and fails to predict aortic pressure in the systolic phase. In fact, the frequency analysis of the aortic input impedance pattern shows that the complex modulus decreases to a negligible value and the phase angle reaches $-90^{\circ}$ at medium and high frequency. This response is in contrast with that measured in-vivo, where the modulus reduces to a plateau value and the phase angle tends to $0^{\circ}$ [3] [13]. In order to overcome this weakness, a three-element Windkessel (3-WK) model has been proposed. Compared to $2-\mathrm{WK}$, the latter model adds a characteristic impedance $Z_{C}$ connected in series to WK2 block, as shown in Fig 1, which helps to improve the frequency response and provides a realistic shape of the blood flow and aortic pressure [14]. Since the pioneer Frank's model, several modified Windkessel configurations have been suggested with the objective to improve the accuracy of arterial hemodynamic prediction. The models varied in the numbers of electrical elements used to represent the arterial system variables [6]. Although, 3-WK and the latest Windkessel modified model were able to produce an acceptable hemodynamic characterization, many research papers claimed that the compliance $C$ which represents the elasticity ability of the blood vessel, was not well estimated. Indeed, these models considered that the arterial vessel is a pure elastic reservoir (represented by an ideal capacitor) which contrasts the natural behavior. In fact, similar to the

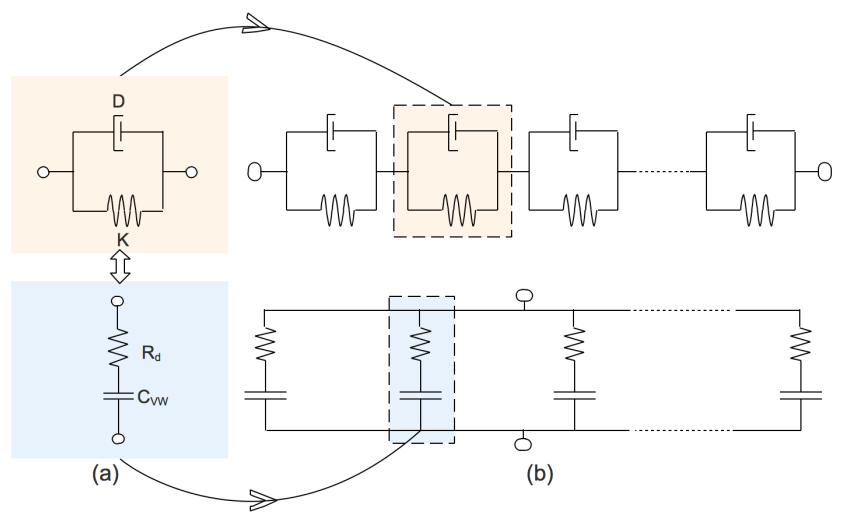

Fig. 2: (a) Viscoelastic Voigt Cell model consisting of a spring with elastic constant $\mathrm{K}$ connected in parallel to a dashpot with a damping factor $\mathrm{D}$ and its electrical analogue that consists of a resistor $\mathrm{R}_{d}$ in series with a capacitor $\mathrm{C}_{v w}$ and (b) Viscoelastic ladder network comprising an infinite Voigt cells connected in series and its electrical analogue.

most existing biological tissues, vascular vessel wall shows a viscoelastic property rather than pure elastic one [4].

The transmission of the mechanical energy through arteries might be split into two modules: the first module presents the stored energy into wall tissues that can be retrieved due to elasticity capabilities, while the second module is dissipated due to the viscosity property [15]. Hence, some research attempts have tried to characterize this behavior by developing Viscoelastic Windkessel (VWK) Models. Those representations are similar to the standard WK; however, the ideal capacitor $C$ is replaced by a complex and frequency dependent capacitor. This latter complex compliance is developed based on the mechanical Voigt cell model whose electrical analogous is a resistor $R_{d}$ in series with a capacitor $C_{v w}$ representing respectively the viscous and elastic phenomena, as illustrated in Fig. 2 (a). However, Roberto et al. [5] showed that modeling the arterial vessel motion using Voigt cell model is limited and does not certainly yield to a realistic representation of the vascular viscoelasticity. This is because, Voigt characterization does not account for the stress-relaxation experiment [16]. This issue can be addressed by increasing the viscoelastic model order as illustrated by the ladder network in Fig. 2b, however, with the ladder model the number of parameters to identify is very large which define another challenge. Literally, the collected data based physiological aortic input impedance is deemed insufficient to identify all the variables for these complex models. In addition, it is well known that reduced order models are preferable for their simplicity and their ease to characterize and use.

Recently, several studies proved that the arterial viscoelasticity exhibits fractional order behavior. Accordingly, the study of vascular wall motion within fractional modeling framework may overcome the previously mentioned inconsistencies. In fact, fractional order models may provide good behavior with less parameters. In addition, the representation of arterial viscoelasticity using ladder structure can be replaced simply by a two-element fractional order model with that is able to capture accurately the real behavior [17]. 

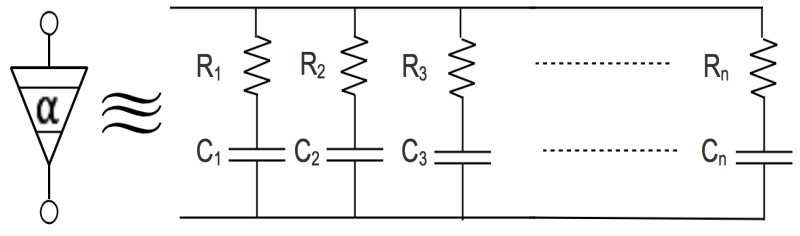

Fig. 3: RC network for fractional order capacitor emulation.

\section{B. Fractional Derivative}

Fractional derivative (FD) is a generalization of the traditional integer derivative to non-integer order. The interest in FD has grown considerably and it is now used in many fields of engineering and science. Due to its non-locality and memory properties, FD is a powerful tool for modeling physical phenomena involving memory effect or delays. Many studies have investigated modeling and analyzing biomedical and biological systems using fractional calculus [18]. In addition to the physiological insights that these fractional models provide, the differentiation orders offer new parameters that allow capturing more general behaviors that the integer order models fail to describe accurately and with less equations. Bearing these wider capabilities in mind, several research studies have shown that FD is an ideal tool to characterize the viscoelasticity property of different biological tissues.

The biomechanical behavior of the arteries is determined by its three main wall components: smooth muscle fibers, elastin fibers, and collagen fibers. Vascular activation is defined as the smooth muscle fiber stretching action on the collagenous fiber [19]. Scientists have noticed that this activity affects the local viscoelastic behavior of the vessels. Thus, we have modulating viscoelasticity that lead to nonlocality property. Recently, Caraiem et al. [8]-[10] proposed a fractional order model to describe the response of the aorta viscoelasticity. They showed that the fractional differentiation order value $(\alpha)$ can be associated to the arterial modulating viscoelasticity, smooth muscles action. In order to validate their model, stress-relaxation experiment have been used.

According to Reimann and Liouville definition, the fractional order derivative $\alpha$ of a function $\mathrm{g}(\mathrm{t})$ can be formulated [20] as follow:

$$
D^{\alpha} g(t)=\frac{1}{\Gamma(1-\alpha)} \frac{\mathrm{d}}{\mathrm{d} t} \int_{0}^{t} \frac{g(\tau)}{(1-\tau)^{\alpha}} d \tau
$$

where $\Gamma$ is the Euler gamma function. Assuming null initial conditions for simplicity, in the Laplace domain, the fractional operator is given by:

$$
D^{\alpha} g(t) \stackrel{L}{\rightarrow} s^{\alpha} G(s),
$$

where $s$ is the Laplace variable.

\section{Method And Model}

In the systemic arterial system, a lumped parametric model is an analog circuit arrangement that leads to the same input impedance as the arterial network disposes, in a specific frequency domain [21]. In this section, we present a threeelement based fractional-order arterial model. The proposed model has a similar configuration as 3-WK. However, it

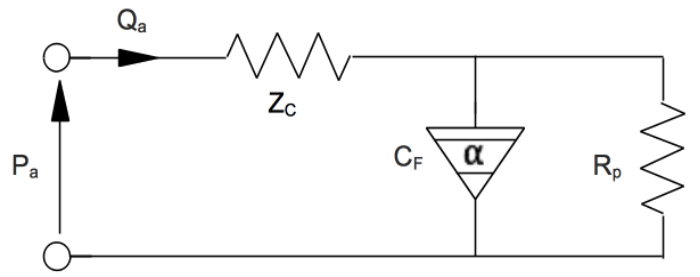

Fig. 4: Three element Fractional-Order Viscoelastic Windkessel model analog circuit. This model consists of total peripheral resistance, $R_{p}$ in parallel to a fractional-order capacitor $C_{F}$ connected in series to the aortic characteristic impedance $Z_{C}$.

incorporates a fractional-order capacitor rather than an ideal one. The non-ideal component presents the fractional arterial viscoelasticity behavior.

\section{A. Fractional order capacitor}

A fractional order capacitor is an electrical component which consists of two parallel plates confining a loss material. It can also be defined as a constant phase element (CPE) whose equivalent impedance has a constant phase angle (between $0^{\circ}$ and $-90^{\circ}$ ) over the whole frequency band, from zero to infinity [22] [23].

The well-known relationship linking the current $i_{c}$ flowing through an ideal capacitor $C$ and the voltage $v_{c}$ between its terminal is as follow:

$$
i_{c}=C \frac{\mathrm{d} v_{c}}{\mathrm{~d} t}
$$

Applying Laplace transformation to Eq. 3 and assuming null initial conditions we obtain:

$$
I_{c}=C s V_{c} \text {. }
$$

The impedance of an ideal capacitor is the ratio of the voltage to the current flowing, in frequency domain. Accordingly, the capacitor's impedance is expressed as:

$$
Z_{C}(s)=\frac{1}{C s} \text {. }
$$

Using the fractional mathematical framework given by Eq. 1 and 2 the Laplace impedance of a fractional-order capacitor $\left(C_{F}\right)$ is:

$$
Z_{C F}(s)=\frac{1}{C_{F} s^{\alpha}},
$$

where $C_{F}$ is the fractional-order capacitance whose Farad $/ \mathrm{sec}^{1-\alpha}$. The Fractional impedance is variable with an exponent $\alpha(0<\alpha<1)$, which is the fractional differentiation order. Many research studies tried to approximate fractional-order capacitors by combining ideal elements in different ways. The most optimized and recognized one is by developing an accurate approximation of the variable $s^{\alpha}$. Several methods have been developed in this direction such us Carlson, Muatsuda, Outsaloup and continued fraction expansion methods [24]-[27]. The latter is the most attractive. In fact, it is optimized in term of variables that yield to a minimum gain error and phase angle for $s^{\alpha}$ estimation around a certain frequency $w$ [28]. The corresponding expansion may be written as follow:

$$
s^{n} \approx w^{\alpha} \frac{a_{n} s^{n}+a_{n-1} w s^{n-1}+\ldots+a_{1} s w^{n-1}+a_{0} w^{n}}{b_{n} s^{n}+b_{n-1} w s^{n-1}+\ldots+b_{1} s w^{n-1}+b_{0} w^{n}},
$$


where $n$ represents the approximation order and $w$ the centered frequency where the approximation is performed. Substituting Eq. (7) in (6) we obtain:

$$
Z_{C F}=\frac{1}{C_{F} w} \frac{a_{n} s^{n}+a_{n-1} w s^{n-1}+\ldots+a_{1} s w^{n-1}+a_{0} w^{n}}{b_{n} s^{n}+b_{n-1} w s^{n-1}+\ldots+b_{1} s w^{n-1}+b_{0} w^{n}} .
$$

The fractional order impedance approximation might be implemented using an electrical circuit with a recursive association of resistance and capacitance elements: Foster integer-order ladder $R C$ network as demonstrated in figure 3 [29]. It is worth to notice that this configuration is similar to the viscoelastic analog circuit model represented in Figure 2 (b). Furthermore, for $s=j w$, the value of the fractional capacitor at a specific frequency $w$ will be calculated using this expression:

$$
Z_{C F}(j w)=\underbrace{\frac{1}{C_{F} w^{\alpha}} \cos (\phi)}_{Z_{D}}-\underbrace{j \frac{1}{C_{F} w^{\alpha}} \sin (\phi)}_{Z_{S}},
$$

where $\phi=\alpha \frac{\pi}{2}$. For $\alpha=0, Z_{F C}$ refers to an ideal resistor whose phase angle equals to $0^{\circ}$. For $\alpha=1, Z_{F C}$ remains to an ideal capacitor with a phase angle equal to $-90^{\circ}$.

$$
\left|Z_{C F}\right|=\sqrt{\left(\frac{1}{C_{F} w^{\alpha}} \cos \left(\alpha \frac{\Pi}{2}\right)\right)^{2}+\left(\frac{1}{C_{F} w^{\alpha}} \sin \left(-\alpha \frac{\Pi}{2}\right)\right)^{2}} .
$$

From Eq. (9), $Z_{F C}$ may be split into a dissipative component $Z_{D}$ (represented by the real part) and a storage component $Z_{S}$ (represented by the imaginary part). With analogy to mechanical behavior, these terms lead to exhibit the viscous and elastic part of the arterial vessel wall. Thus, in our study, the fractional element combines both resistive and capacitive properties which displays the fractional viscoelastic behavior of the arterial vessel. The impact of both properties is coordinated by the fractional factor $\alpha$ allowing a real physiological description and decreasing the number of variables.

\section{B. Proposed Model}

Aortic input impedance is a simple characterization of the systemic arterial system. It presents the arterial parameters in a comprehensive way. It is formulated as the ratio between the frequency component of the output arterial blood pressure $\left(P_{a}\right)$ and the input blood flow $\left(Q_{a}\right)$ harmonics, Eq. (12). In reverse, when the aortic input impedance is identified, a given blood flow permits the control of the arterial pressure and vice versa.

$$
Z_{\text {in }}=\frac{P_{a}}{Q_{a}} .
$$

The proposed model is shown in Fig 4. Applying Kirchhoff's Voltage law (KVL) to the left mesh of the circuit, the voltage
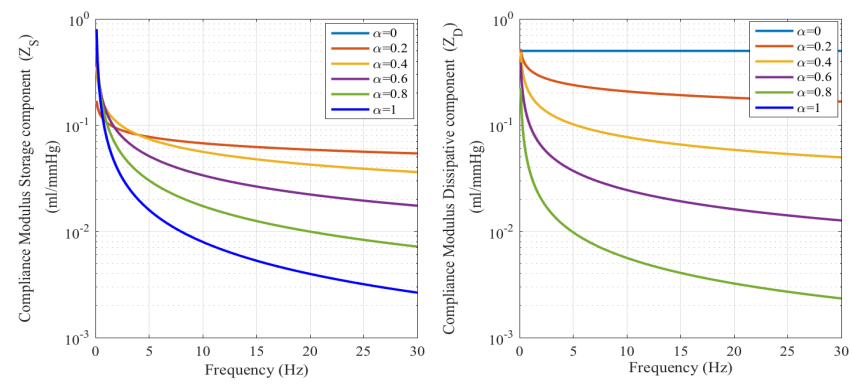

Fig. 5: Storage modulus $Z_{S}$ and Dissipation modulus $Z_{D}$ of the complex arterial compliance for different fractional differentiation order $\alpha$.

between the terminals of the fractional order capacitor can be expressed as follow:

$$
v_{C F}(t)=P_{a}(t)-Z_{C} Q_{a}(t) .
$$

Applying Kirchhoff's Current law (KCL) we obtain:

$$
Q_{a}(t)=C_{F} D_{\alpha}^{t} v_{C F}(t)+\frac{v_{C F}(t)}{R_{p}} .
$$

Substituting Eq. 12 in 13 gives the following equations.

$$
\begin{aligned}
& Q_{a}(t)=C_{F} D_{\alpha}^{t}\left(P_{a}(t)-Z_{C} Q_{a}(t)\right)+\frac{\left(P_{a}(t)-Z_{C} Q_{a}(t)\right)}{R_{p}}, \\
& Z_{C} C_{F} D_{\alpha}^{t} Q_{a}(t)+Q_{a}(t)\left(1+\frac{Z_{C}}{R_{p}}\right)=C_{F} D_{\alpha}^{t} P_{a}(t)+\frac{P_{a}(t)}{R_{p}}
\end{aligned}
$$

Applying Laplace transformation and assuming null initial conditions, the fractional aortic input impedance $Z_{F}$ may be expressed as:

$$
Z_{F}(s)=\left(R_{p}+Z_{C}\right) \frac{1+s^{\alpha} \tau_{N}}{1+s^{\alpha} \tau_{D}},
$$

where $\tau_{N}$ and $\tau_{D}$ are time constants and have the following expressions:

$$
\left\{\begin{array}{c}
\tau_{N}=\frac{R_{p} Z_{C}}{R_{p}+Z_{C}} C_{F}, \\
\tau_{D}=R_{p} C_{F} .
\end{array}\right.
$$

Thus the input impedance modulus and phase angle at a specific frequency $w$ is given by Eq. (18) and (19).

\section{Simulation Results and Discussion}

In this section, we provide a comprehensive analysis of the previous derived model, taking into consideration the fractional-order parameter $\alpha$. We study the effect of varying the fractional differentiation order value $\alpha$ on the complex and frequency dependent arterial compliance and arterial stiffness. We also examine the variation of aortic input impedance modulus and phase angle for the developed model versus $\alpha$. For the simulation, we use MATLAB_R2016b and all the parameters values of the systemic arterial system

$$
\begin{gathered}
\left|Z_{F}\right|=\left(R_{p}+Z_{C}\right) \frac{\sqrt{\left(1+w^{\alpha} \tau_{N} \cos \left(\alpha \frac{\pi}{2}\right)\right)^{2}+\left(w^{\alpha} \tau_{N} \sin \left(\alpha \frac{\pi}{2}\right)\right)^{2}}}{\sqrt{\left(1+w^{\alpha} \tau_{D} \cos \left(\alpha \frac{\pi}{2}\right)\right)^{2}+\left(w^{\alpha} \tau_{D} \sin \left(\alpha \frac{\pi}{2}\right)\right)^{2}}} \\
\angle Z_{F}=\tan ^{-1}\left(\frac{w^{\alpha} \tau_{N} \sin \left(\alpha \frac{\pi}{2}\right)}{\left.1+w^{\alpha} \tau_{N} \cos \left(\alpha \frac{\pi}{2}\right)\right)}\right)-\tan ^{-1}\left(\frac{w^{\alpha} \tau_{D} \sin \left(\alpha \frac{\pi}{2}\right)}{\left.1+w^{\alpha} \tau_{D} \cos \left(\alpha \frac{\pi}{2}\right)\right)}\right) .
\end{gathered}
$$



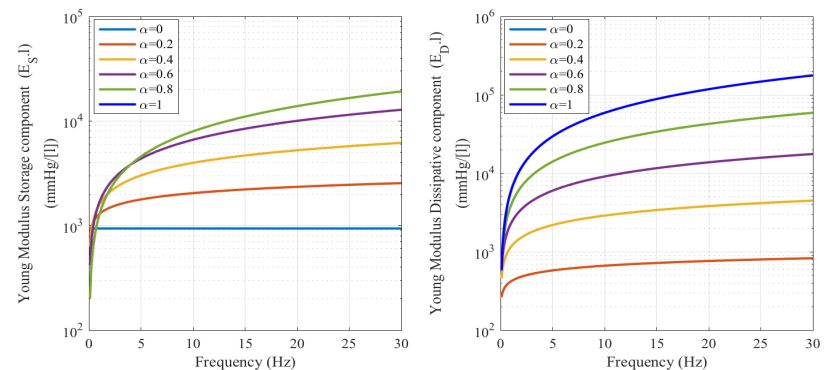

Fig. 6: Storage modulus $E_{S}$ and Dissipation modulus $E_{D}$ of the complex Young modulus for different fractional differentiation order $\alpha$.

TABLE I: Arterial Parameter Based Three-Element Windkessel Model

\begin{tabular}{llll}
\hline Parameter (dimensions) & Hypotensive & Normotensive & Hypertensive \\
\hline \hline$Z_{C}(\mathrm{mmHg} . \mathrm{s} / \mathrm{ml})$ & 0.02 & 0.033 & 0.05 \\
$C(\mathrm{ml} / \mathrm{mmHg})$ & 2 & 1.5 & 0.7 \\
$R_{p}(\mathrm{mmHg} . \mathrm{s} / \mathrm{ml})$ & 0.6 & 0.95 & 1.4 \\
\hline
\end{tabular}

were taken from [14] [30] [31] which represent the standard three element Windkessel model variables in normal and pathological conditions as shown in Table I.

\section{A. Effect of $\alpha$ on the total arterial Stiffness}

Arterial stiffness $(A S)$ is a key metric for the evaluation of the degenerative diseases physio-pathological mechanisms that affect CVS. $A S$ characterizes the vessel material properties and is measured based on the Young's Modulus $E$. In clinical practice, we cannot measure directly $E$ because isolating the arterial tissue without perfusion is not feasible. Hence, we usually use the pressure-volume and pressureradius blood vessel relationships which define the two wellknown parameters, the total complex and frequency dependent arterial Compliance $\left(C_{a}\right)$ and Elastance $\left(E_{a}\right)$. The last two metrics are defined as the aptitude of the blood vessel to contract and expand with the variation of the blood pressure [21]. The Arterial Compliance $C_{a}$ is the inverse of $E_{a}$ and expressed as follow:

$$
C_{a}=\frac{1}{E_{a}}=\frac{\Delta V}{\Delta P} .
$$

In order to estimate the Young Modulus $E$, we usually use the following relation that links $E$ to $C$ proposed by Love [32]:

$$
C_{a} \approx \frac{3 \Pi l r_{i}^{2}}{2 E h},
$$

where $r_{i}$ is the internal artery radius, $h$ is the vessel wall thickness and $l$ is the arterial length. Fig. 5 and 6 show the variation of the real and imaginary parts of the fractional complex and frequency dependent compliance $Z_{C F}$ and $E$. As the fractional-order parameter $\alpha$ increases from 0 to 1 , implying an increase in the wall level elasticity, the storage and dissipative modulus of the compliance increases, however that of Young Modulus decreases across all the frequencies [33].

\section{B. Effect of $\alpha$ on the aortic input Impedance}

As revealed previously, the aortic input impedance represents a simple and comprehensive resource for hemodynamic parameters evaluation and vascular pathologies identifications. In addition, from of the aortic input impedance

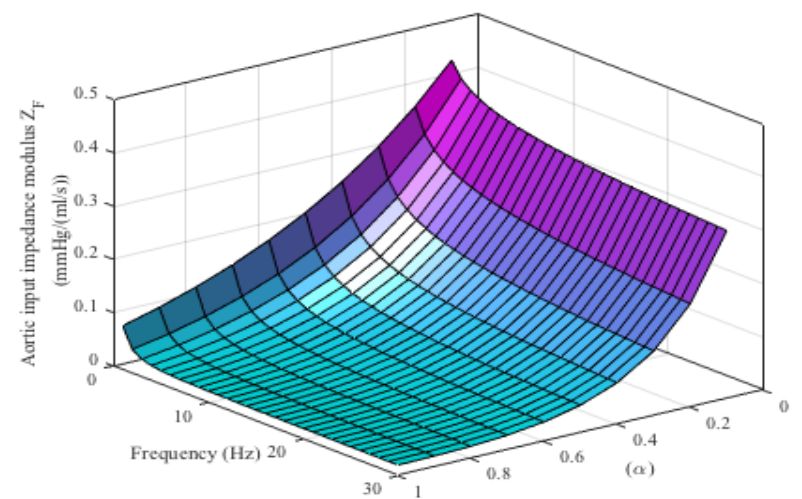

Fig. 7: Numerical simulation of the aortic input impedance versus the fractional differentiation order $\alpha$ and frequency.

modulus plot we can easily extract the three-fundamental arterial parameters: total peripheral resistance $R_{p}$, total arterial Compliance $C$ and the aortic Characteristic impedance $Z_{C}$. Hence, get a sufficient idea about the arterial system state. Fig. 7 presents the variation of the aortic input impedance modulus versus the differentiation order $\alpha$ across frequencies. It is clear that changing $\alpha$ which means varying the degree of vessel wall viscosity influences all the hemodynamic parameters. Physiologically, it is known that the variation of the vessel wall resistivity contributes to the development and genesis of systemic arterial system deceases such as hypertensive (high blood pressure) and hypo-tensive) low blood pressure. As an investigation, we plot in Fig 8, the modulus and phase angle of a normo-tensive aortic input impedance for different differentiation orders $\alpha$ together with hypertensive and hypo-tensive input impedance. By tuning $\alpha$ that implies changing in vessel resistivity, conduct the arterial system from normo-tensive to hypertensive or hypotensive. Thus, the arterial viscoelastic order described by $\alpha$
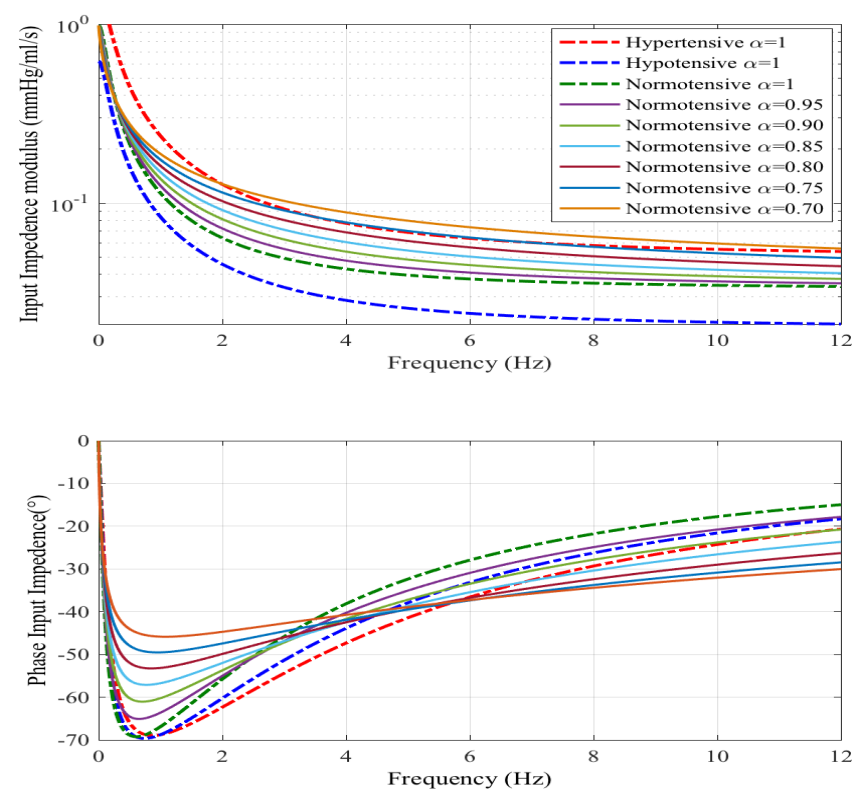

Fig. 8: Example of modulus and phase angle of aortic input impedance for different fractional differentiation order $\alpha$ plotted together with normotensive, hypo-tensive and hypertensive aortic input impedance modulus and phase using standard three-element Windkessel model. 
could contribute to understand the mechanism through which the arterial system hemodynamic behavior changes with the changes of vessel tissue properties.

\section{CONCLUSION}

Cardiovascular system wall tissues show a viscoelastic behavior rather than pure elastic. In addition, arteries which constitute the major part of the arterial network should be considered as a viscoelastic reservoir due to the superposition of viscous and elastic components. Recently, many studies have shown that the arterial wall mechanics can be described using fractional order models, reducing the number of parameters and showing a natural response. In the presented work, we proposed a three-element fractional order viscoelastic Windkessel model to describe the arterial hemodynamic. We believe that the deep understanding of arterial viscoelastic behavior is of paramount importance in the study of physio pathological mechanisms of degenerative disease affecting CVS. Although, all the simulation results are based on validated physiological parameters, a limitation of this work that one may argue, is no experimental data has been investigated. In this paper, we just study the contribution of both arterial viscous and elastic properties controlled by the fractional differentiation order $\alpha$. Nevertheless, ongoing work includes the valiation of proposed model experimentally.

\section{ACKNOWLEDGMENT}

Research reported in this publication was supported by King Abdullah University of Science and Technology (KAUST).

\section{REFERENCES}

[1] F. N. Van de Vosse and N. Stergiopulos, "Pulse wave propagation in the arterial tree," Annual Review of Fluid Mechanics, vol. 43, pp. 467-499, 2011.

[2] I. Kokalari, T. Karaja, and M. Guerrisi, "Review on lumped parameter method for modeling the blood flow in systemic arteries," Journal of Biomedical Science and Engineering, vol. 6, no. 01, p. 92, 2013.

[3] N. Westerhof, J.-W. Lankhaar, and B. E. Westerhof, "The arterial windkessel," Medical \& biological engineering \& computing, vol. 47, no. 2, pp. 131-141, 2009.

[4] R. Gul, "Mathematical modeling and sensitivity analysis of lumpedparameter model of the human cardiovascular system," Ph.D. dissertation, Freie Universität Berlin, 2016.

[5] R. Burattini and S. Natalucci, "Complex and frequency-dependent compliance of viscoelastic windkessel resolves contradictions in elastic windkessels," Medical engineering \& physics, vol. 20, no. 7, pp. 502514, 1998.

[6] M. Capoccia, "Development and characterization of the arterial windkessel and its role during left ventricular assist device assistance," Artificial organs, vol. 39, no. 8, 2015.

[7] T. C. Doehring, A. D. Freed, E. O. Carew, and I. Vesely, "Fractional order viscoelasticity of the aortic valve cusp: an alternative to quasilinear viscoelasticity," Journal of biomechanical engineering, vol. 127, no. 4, pp. 700-708, 2005.

[8] D. Craiem and R. L. Armentano, "A fractional derivative model to describe arterial viscoelasticity," Biorheology, vol. 44, no. 4, pp. 251263, 2007.

[9] D. Craiem, F. Rojo, J. Atienza, G. Guinea, and R. L. Armentano, "Fractional calculus applied to model arterial viscoelasticity," Latin American applied research, vol. 38, no. 2, pp. 141-145, 2008.

[10] D. Craiem, F. J. Rojo, J. M. Atienza, R. L. Armentano, and G. V. Guinea, "Fractional-order viscoelasticity applied to describe uniaxial stress relaxation of human arteries," Physics in medicine and biology, vol. 53, no. 17, p. 4543, 2008.
[11] J. P. Zerpa, A. Canelas, B. Sensale, D. B. Santana, and R. Armentano, "Modeling the arterial wall mechanics using a novel high-order viscoelastic fractional element," Applied Mathematical Modelling, vol. 39, no. 16, pp. 4767-4780, 2015.

[12] L. Formaggia and A. Veneziani, "Reduced and multiscale models for the human cardiovascular system," Lecture notes VKI lecture series, vol. 7, 2003.

[13] N. Stergiopulos, J. Meister, and N. Westerhof, "Evaluation of methods for estimation of total arterial compliance," American Journal of Physiology-Heart and Circulatory Physiology, vol. 268, no. 4, pp. H1540-H1548, 1995.

[14] T. Kind, T. J. Faes, J.-W. Lankhaar, A. Vonk-Noordegraaf, and M. Verhaegen, "Estimation of three-and four-element windkessel parameters using subspace model identification," IEEE Transactions on Biomedical Engineering, vol. 57, no. 7, pp. 1531-1538, 2010.

[15] M. Nichelatti, P. Pettazzoni, and G. Pallotti, "The study of viscoelastic behavior of blood vessels," 2017.

[16] J. M. Canty Jr, F. J. Klocke, and R. E. Mates, "Pressure and tone dependence of coronary diastolic input impedance and capacitance," American Journal of Physiology-Heart and Circulatory Physiology, vol. 248, no. 5, pp. H700-H711, 1985.

[17] B. J. West, "Fractal physiology and the fractional calculus: a perspective," Frontiers in physiology, vol. 1, 2010.

[18] R. L. Magin, Fractional calculus in bioengineering. Begell House Redding, 2006.

[19] R. L. Armentano, J. G. Barra, D. B. Santana, F. M. Pessana, S. Graf, D. Craiem, L. M. Brandani, H. P. Baglivo, and R. A. Sanchez, "Smart damping modulation of carotid wall energetics in human hypertension," Hypertension, vol. 47, no. 3, pp. 384-390, 2006.

[20] G. Jumarie, "Modified riemann-liouville derivative and fractional taylor series of nondifferentiable functions further results," Computers \& Mathematics with Applications, vol. 51, no. 9-10, pp. 1367-1376, 2006.

[21] N. Westerhof, N. Stergiopulos, and M. I. Noble, "Arterial input impedance," in Snapshots of Hemodynamics. Springer, 2010, pp. 161-171.

[22] A. S. Elwakil, "Fractional-order circuits and systems: An emerging interdisciplinary research area," IEEE Circuits and Systems Magazine, vol. 10, no. 4, pp. 40-50, 2010.

[23] C. M. Ionescu, J. T. Machado, and R. De Keyser, "Modeling of the lung impedance using a fractional-order ladder network with constant phase elements," IEEE Transactions on biomedical circuits and systems, vol. 5, no. 1, pp. 83-89, 2011.

[24] G. Carlson and C. Halijak, "Approximation of fractional capacitors $(1 / \mathrm{s})^{\wedge}(1 / \mathrm{n})$ by a regular newton process," IEEE Transactions on Circuit Theory, vol. 11, no. 2, pp. 210-213, 1964.

[25] Y. Q. Chen and K. L. Moore, "Discretization schemes for fractionalorder differentiators and integrators," IEEE Transactions on Circuits and Systems I: Fundamental Theory and Applications, vol. 49, no. 3, pp. 363-367, 2002.

[26] A. Oustaloup, F. Levron, B. Mathieu, and F. M. Nanot, "Frequencyband complex noninteger differentiator: characterization and synthesis," IEEE Transactions on Circuits and Systems I: Fundamental Theory and Applications, vol. 47, no. 1, pp. 25-39, 2000.

[27] G. Tsirimokou, "A systematic procedure for deriving rc networks of fractional-order elements emulators using matlab," AEU-International Journal of Electronics and Communications, vol. 78, pp. 7-14, 2017.

[28] B. Krishna and K. Reddy, "Active and passive realization of fractance device of order 1/2," Active and passive electronic components, vol. 2008, 2008.

[29] J. Machado, "Discrete-time fractional-order controllers," Fractional Calculus and Applied Analysis, vol. 4, pp. 47-66, 2001.

[30] N. Stergiopulos, B. E. Westerhof, and N. Westerhof, "Total arterial inertance as the fourth element of the windkessel model," American Journal of Physiology-Heart and Circulatory Physiology, vol. 276, no. 1, pp. H81-H88, 1999.

[31] P. Segers, N. Stergiopulos, and N. Westerhof, "Quantification of the contribution of cardiac and arterial remodeling to hypertension," Hypertension, vol. 36, no. 5, pp. 760-765, 2000.

[32] A. E. H. Love, A treatise on the mathematical theory of elasticity. Cambridge university press, 2013.

[33] H. Xiao, I. Tan, M. Butlin, D. Li, and A. P. Avolio, "Arterial viscoelasticity: role in the dependency of pulse wave velocity on heart rate in conduit arteries," American Journal of Physiology-Heart and Circulatory Physiology, vol. 312, no. 6, pp. H1185-H1194, 2017. 